\title{
DIFFERENTIAL INEQUALITIES ON HOMOGENEOUS TORIC BUNDLES
}

\author{
AN-MIN LI, LI SHENG, AND GUOSONG ZHAO
}

\begin{abstract}
We study a generalized Abreu Equation in $n$-dimensional polytopes and prove some differential inequalities for homogeneous toric bundles.
\end{abstract}

Keywords. generalized Abreu Equation, differential inequalities. MSC[2008] 53C55 35J60

\section{INTRODUCTION}

The existence of extremal and contant scalar curvature is a central problem in Kähler geometry. In a series of papers [11], [12], [13], and [14], Donaldson studied this problem on toric manifolds and proved the existence of metrics of constant scaler curvatures on toric surfaces under an appropriate stability condition. Later on in [7] and [8], Chen, $\mathrm{Li}$ and Sheng proved the existence of metrics of prescribed scaler curvatures on toric surfaces under the uniform stability condition.

It is important to generalize the results of Chen, $\mathrm{Li}$ and Sheng to more general Kähler manifold. This is one of a sequence of papers, aiming at generalizing the results of Chen, $\mathrm{Li}$ and Sheng to homogeneous toric bundles. A homogeneous toric bundle $G \times_{K} M$ have a compact toric manifold $M$ as fiber and a generalized flag manifold $G / K$ as basis. Let $U \subset M$ be any complex coordinate domain and let $G(U) \subset G \times_{K} M$ be the image of $U$ under the left $G$-action on $G \times_{K} M$. In this paper we establish some differential inequalities on $G(U)$, which are generalizations of the differential inequalities in [7]. The differential inequalities of this paper play a important role in our works on scaler curvatures for homogeneous toric bundles.

\section{HOMOGENEOUS TORIC BUNDLES}

We recall some facts about homogeneous toric bundles and refer to [20], [4] and [15] for details. Let $G$ be a compact semisimple Lie group, $K$ be the centralizer of a torus $S$ in $G$. Let $T$ be a maximal torus in $G$ containing $S$. Then $T \subset C(S)=K, G / K$ is a generalized flag manifold. Denote $\mathfrak{o}=e K$. Let $\mathfrak{g}$ (resp. $\mathfrak{k}, \mathfrak{h}$ ) be the Lie algebra of $G$

Li acknowledges the support of NSFC Grant 11521061.

Sheng acknowledges the support of NSFC Grant 11471225.

Zhao acknowledges the support of NSFC Grants 11571242. 
(resp. $K, T$ ). Let $B$ denote the Killing form of $\mathfrak{g}$. Recall that $-B$ is a positive definite inner product on $\mathfrak{g}$. There is a orthogonal decomposition with respect to $-B$ :

$$
\mathfrak{g}=\mathfrak{k} \oplus \mathfrak{m}, \quad \operatorname{Ad}(k) \mathfrak{m} \subset \mathfrak{m} \text { for all } k \in K .
$$

The tangent space of $G / K$ at $\mathfrak{o}$ is identified with $\mathfrak{m}$.

Let $Z(K)$ be the center of $K$, it is an n-dimensional torus, denoted by $T^{n}$. Let $(M, \omega)$ be a compact toric Kähler manifold of complex dimension $n$, where $T^{n}$ acts effectively on $M$. Let $\varrho: K \rightarrow T^{n}$ be a surjective homomorphism. The homogeneous toric bundle $G \times{ }_{K} M$ is defined to be the space $G \times M$ modulo the relation $(g h, x)=(g, \varrho(h) x)$, where $g \in G, h \in K$, and $x \in M$. Later we will omit $\varrho$ to simplify notations. The space $G \times{ }_{K} M$ is a fiber bundle with fiber $M$ and base space $G / K$, a generalized flag manifold. There is a natural $G$-action on $G \times{ }_{K} M$ given by $g \cdot[h, x]=[g h, x], g \in G, x \in M$, and a natural $T^{n}$-action on $G \times{ }_{K} M$ given by $k \cdot[g, x]=\left[g, k^{-1} x\right], k \in T^{n}$. Both $G / K$ and $G \times{ }_{K} M$ are complex manifolds. In fact, they can be expressed as $G^{\mathbb{C}} / P$ and $G^{\mathbb{C}} \times{ }_{P} M$ respectively, where $G^{\mathbb{C}}$ is the complexification of $G, P$ is a parabolic subgroup of $G^{\mathbb{C}}$.

Denote by $\mathfrak{g}^{\mathbb{C}}, \mathfrak{k}^{\mathbb{C}}, \mathfrak{h}^{\mathbb{C}}$ the complexification of $\mathfrak{g}, \mathfrak{k}, \mathfrak{h}$ respectively. Let $R$ be the root system of $\mathfrak{g}^{\mathbb{C}}$ with respect to $\mathfrak{h}^{\mathbb{C}}$, we have the root space decomposition

$$
\begin{gathered}
\mathfrak{g}^{\mathbb{C}}=\mathfrak{h}^{\mathbb{C}} \oplus \sum_{\alpha \in R} \mathbb{C} E_{\alpha}=\mathfrak{k}^{\mathbb{C}} \oplus \mathfrak{m}^{\mathbb{C}}, \\
\mathfrak{k}^{\mathbb{C}}=\mathfrak{h}^{\mathbb{C}} \oplus \sum_{\alpha \in R_{K}} \mathbb{C} E_{\alpha}, \quad \mathfrak{m}^{\mathbb{C}}=\sum_{\alpha \in R_{M}} \mathbb{C} E_{\alpha},
\end{gathered}
$$

where $R_{K}$ is a subset of $R$ and $R_{M}=R \backslash R_{K}$. For any $\varphi \in \mathfrak{h}^{*}$ we define $h_{\varphi} \in \mathfrak{h}$ by

$$
B\left(h, h_{\varphi}\right)=\varphi(h) \quad \text { for all } h \in \mathfrak{h},
$$

and set

$$
H_{\varphi}=\sqrt{-1} h_{\varphi}
$$

Define

$$
\mathfrak{t}=\mathfrak{h} \bigcap \mathfrak{z}^{\left(\mathfrak{k}^{\mathbb{C}}\right)}
$$

Let

$$
\kappa:\left.\mathfrak{h}^{*} \rightarrow \mathfrak{t}^{*} \quad \alpha \mapsto \alpha\right|_{\mathfrak{t}}
$$

be the restriction map. Set $R_{T}=\kappa(R)=\kappa\left(R_{M}\right)$. The elements of $R_{T}$ are called $T$-roots.

Choose a fundamental system $\Pi=\left\{\alpha_{1}, \cdots, \alpha_{n}, \alpha_{n+1}, \cdots, \alpha_{n+\hbar}\right\}$ of $R$. We fix a lexicographic ordering and let $R^{+}$(resp. $R_{K}^{+}$) be the set of positive roots of $R$ ( resp. 
$R_{K}$ ) with respect to $\Pi$. Let $R_{M}^{+}=R^{+} \backslash R_{K}^{+}$. Denote $\Pi_{K}=\Pi \cap R_{K}$. We assume that $\Pi_{K}=\left\{\alpha_{n+1}, \cdots, \alpha_{n+\hbar}\right\}$. For $i \leq n$, let

$$
\tilde{h}_{\alpha_{i}}=h_{\alpha_{i}}+\sum_{j=n+1}^{n+\hbar} d_{i}^{j} h_{\alpha_{j}}
$$

where $d_{i}^{j}$ are constants such that $B\left(\tilde{h}_{\alpha_{i}}, h_{\alpha_{j}}\right)=0$ for all $j=n+1, \cdots, n+\hbar$. Denote

$$
H_{\alpha_{i}}=\sqrt{-1} h_{\alpha_{i}}, \quad i=n+1, \cdots, n+\hbar, \quad \tilde{H}_{\alpha_{j}}=\sqrt{-1} \tilde{h}_{\alpha_{j}}, \quad j=1, \cdots, n .
$$

For any $\alpha \in R_{K}$, we have $\alpha\left(\tilde{H}_{\alpha_{j}}\right)=0$. Then $\mathfrak{t}=\operatorname{span}\left\{\tilde{H}_{\alpha_{1}}, \cdots, \tilde{H}_{\alpha_{n}}\right\}$. Let $\mathfrak{h}^{\prime}=$ $\operatorname{span}\left\{H_{\alpha_{n+1}}, \cdots, H_{\alpha_{n+\hbar}}\right\}$. We have the orthogonal decomposition with respect to $-B$ : $\mathfrak{h}=\mathfrak{t} \oplus \mathfrak{h}^{\prime}$.

We choose a Weyl basis $e_{\alpha} \in \mathfrak{g}_{\alpha}^{\mathbb{C}}$ of $\mathfrak{g}^{\mathbb{C}}$ such that, for $e_{\alpha} \in \mathfrak{g}_{\alpha}$ and $e_{-\alpha} \in \mathfrak{g}_{-\alpha}$,

$$
B\left(e_{\alpha}, e_{-\alpha}\right)=1, \quad\left[e_{\alpha}, e_{-\alpha}\right]=h_{\alpha},
$$

Obviously, $\left[h, e_{\alpha}\right]=\alpha(h) e_{\alpha}$, for any $h \in \mathfrak{t}$. Set

$$
V_{\alpha}=e_{\alpha}-e_{-\alpha}, \quad W_{\alpha}=\sqrt{-1}\left(e_{\alpha}+e_{-\alpha}\right), \quad H_{\alpha}=\sqrt{-1} h_{\alpha} .
$$

It is easy to see that $H_{\alpha}, V_{\alpha}, W_{\alpha} \in\left(\mathfrak{g}_{\alpha} \oplus \mathfrak{g}_{-\alpha} \oplus\left[\mathfrak{g}_{\alpha}, \mathfrak{g}_{-\alpha}\right]\right) \cap \mathfrak{g}$. For any $\alpha \in R^{+}$we have $\alpha=\sum_{j=1}^{n+\hbar} M_{\alpha}^{j} \alpha_{j}$ with $M_{\alpha}^{j} \geq 0$. It is easy to see that

$$
H_{\alpha}=\sum_{j=1}^{n+\hbar} M_{\alpha}^{j} H_{\alpha_{j}}=\sum_{j=1}^{n} M_{\alpha}^{j} \tilde{H}_{\alpha_{j}}+\sum_{j=n+1}^{n+\hbar} M_{\alpha}^{\prime j} H_{\alpha_{j}},
$$

where $M_{\alpha}^{\prime j}=M_{\alpha}^{j}-\sum_{i=1}^{n} M_{\alpha}^{i} d_{i}^{j}$. Obviously, for any $\alpha \in R_{M^{+}}$, we have

$$
\sum_{j=1}^{n} M_{\alpha}^{j}>0
$$

\section{3. $\left(G, T^{n}\right)$-INVARIANT KÄHLER METRICS}

From now on, our convention for the ranges of indices is the following:

$$
\begin{gathered}
1 \leq A, B, \ldots \leq n+l \\
1 \leq i, j, k, \ldots \leq n \\
n+1 \leq \alpha \leq n+l
\end{gathered}
$$


where $l$ is the dimension of $\mathfrak{m}$. For any $1 \leq j \leq n, \alpha \in R_{M}^{+}$, let $\tilde{H}_{\alpha_{j}}^{*}, V_{\alpha}^{*}, W_{\alpha}^{*}$ be the fundamental vector fields corresponding to $\tilde{H}_{\alpha_{j}}, V_{\alpha}, W_{\alpha}$. Then the left-invariant vector fields $\left\{\frac{\partial}{\partial x^{j}}, \tilde{H}_{\alpha_{j}}^{*}, V_{\alpha}^{*}, W_{\alpha}^{*}\right\}_{1 \leq j \leq n, \alpha \in R_{M}^{+}}$is a local base of $G \times_{K} M$. Obviously,

$$
\left[\frac{\partial}{\partial x^{j}}, \tilde{H}_{j}^{*}\right]=0, \quad\left[\frac{\partial}{\partial x^{j}}, V_{\alpha}^{*}\right]=\left[\frac{\partial}{\partial x^{j}}, W_{\alpha}^{*}\right]=0, \quad 1 \leq j \leq n, \alpha \in R_{M^{+}} .
$$

Let $\left\{d x^{j}, \nu^{j}, d V^{\alpha}, d W^{\alpha}\right\}_{1 \leq j \leq n, \alpha \in R_{M}^{+}}$be the dual left-invariant 1-form of the base. Set

$$
\begin{aligned}
S_{j}=\frac{1}{2}\left(\frac{\partial}{\partial x^{j}}-\sqrt{-1} H_{j}^{*}\right), & 1 \leq j \leq n \\
S_{\alpha}=\frac{1}{2}\left(V_{\alpha}^{*}-\sqrt{-1} W_{\alpha}^{*}\right), & \alpha \in R_{M^{+} .}
\end{aligned}
$$

We define the almost complex structure $J$ by,

$$
J S_{i}=\sqrt{-1} S_{i}, \quad J S_{\alpha}=\sqrt{-1} S_{\alpha}, \quad \forall 1 \leq i \leq n, \quad \alpha \in R_{M^{+}} .
$$

Then $J$ is a $\left(G, T^{n}\right)$-invariant complex structure. It is easy to see that $S_{j}, S_{\alpha}$ are $(1,0)$ vector fields. Denote $\omega^{j}, \omega^{\alpha}$ the dual $(1,0)$-form of $S_{j}, S_{\alpha}$. It is easy to see that $S_{\alpha}, \bar{S}_{\alpha}$ is induced vector field of $e_{\alpha}$ and $-e_{-\alpha}$. By (2.1) we have

$$
\begin{aligned}
& {\left[S_{j}, \bar{S}_{k}\right]=\left[S_{j}, S_{k}\right]=0, \quad\left[S_{\alpha}, \bar{S}_{\alpha}\right]=-\sqrt{-1} H_{\alpha}^{*}=\sum_{j} M_{\alpha}^{j}\left(S_{j}-\bar{S}_{j}\right),} \\
& {\left[S_{j}, S_{\alpha}\right]=-\frac{1}{2} \alpha\left(\tilde{h}_{j}\right) S_{\alpha}, \quad\left[S_{j}, \bar{S}_{\alpha}\right]=\frac{1}{2} \alpha\left(\tilde{h}_{j}\right) \bar{S}_{\alpha}, \quad\left[S_{\alpha}, S_{\beta}\right]=-N_{\alpha \beta} S_{\alpha+\beta},} \\
& {\left[S_{\alpha}, \bar{S}_{\beta}\right]=N_{\alpha-\beta} S_{\alpha-\beta}, \quad \alpha>\beta \quad\left[S_{\alpha}, \bar{S}_{\beta}\right]=-N_{\alpha-\beta} \bar{S}_{\beta-\alpha}, \quad \alpha<\beta .}
\end{aligned}
$$

As in [19] one can check that

$$
d \nu^{j}=\sum_{\alpha \in R_{M^{+}}} 2 M_{\alpha}^{j} d V^{\alpha} \wedge d W^{\alpha}=\sum_{\alpha \in R_{M^{+}}} M_{\alpha}^{j} \sqrt{-1} \omega^{\alpha} \wedge \bar{\omega}^{\alpha} .
$$

In fact, we have

$$
d \nu^{j}\left(S_{A}, \bar{S}_{B}\right)=S_{A}\left(\nu^{j}\left(\bar{S}_{B}\right)\right)-\bar{S}_{B}\left(\nu^{j}\left(S_{A}\right)\right)-\nu^{j}\left(\left[S_{A}, \bar{S}_{B}\right]\right)=-\nu^{j}\left(\left[S_{A}, \bar{S}_{B}\right]\right) .
$$

Then (3.5) follows from (3.2)-(3.4).

Denote by $\tau: M \rightarrow \bar{\Delta} \subset \mathfrak{t}^{*}$ the moment map of $M$, where $\Delta$ is a Delzant polytope. The left invariant 1 -form $\left\{\nu^{1}, \cdots, \nu^{n}\right\}$ can be seen as a basis of $\mathfrak{t}^{*}$. The moment map $\tau: M \rightarrow \mathfrak{t}^{*}$ has components, relative to this basis of $\mathfrak{t}^{*}$, which we denote by $\tau_{i}$. Note that $\sum_{i=1}^{n} \tau_{i} \nu^{i}$ is independent of the choice of the bases.

Now we fit $G$ into this picture. We fix a point $o \in \mathbb{R}^{n}$ and identify $\mathfrak{t}^{*}$ with $T_{o} \mathbb{R}^{n}$. We choose $\left\{o, \nu^{i}, i=1, \ldots, n\right\}$ as a base of $\mathbb{R}^{n}$. Let $\xi=\left(\xi_{1}, \ldots, \xi_{n}\right)$ be the coordinate system 
with respect to the bases. We choose $\bar{\Delta} \subset\left\{\left(\xi_{1}, \ldots, \xi_{n}\right) \mid \xi_{1}>0, \xi_{2}>0, \ldots, \xi_{n}>0\right\}$ such that

$$
\sum_{\alpha \in R_{M^{+}}} \frac{\sum_{j=1}^{n} M_{\alpha}^{j} \operatorname{diam}(\Delta)}{D_{\alpha}}<\frac{n}{4}
$$

where

$$
D_{\alpha}:=2 \sum_{i=1}^{n} \tau_{i} \nu^{i}=2 \sum_{j=1}^{n} M_{\alpha}^{j} \xi_{j}>0 \quad \forall \xi \in \bar{\Delta} .
$$

Since the moment map is equivariant we can also regard $\tau$ as a map from $G \times{ }_{K} M$ to $\mathfrak{t}^{*}$ and the components $\tau_{i}$ as functions on $G \times{ }_{K} M$, that is, we extend $\tau: G \times_{K} M \rightarrow \bar{\Delta}$ by $\tau([g, x])=\tau(x)$. Following Donaldson ([14]) we consider the following form

$$
\Omega=d\left(\sum_{i=1}^{n} \tau_{i} \nu^{i}\right)
$$

in $\tau^{-1}(\Delta)$. By (3.5) we can write it as

$$
\Omega=\sum_{i=1}^{n} d \tau_{i} \wedge \nu^{i}+\sum_{\alpha \in R_{M^{+}}} D_{\alpha}\left(d V^{\alpha} \wedge d W^{\alpha}\right) .
$$

It is easy to see that $J$ is $\Omega$-compatible. i.e.,

$$
\Omega(X, J X)>0, \quad \forall X \neq 0, \Omega(J X, J Y)=\Omega(X, Y) .
$$

So $\left(G \times_{K} M, \Omega\right)$ is a Kähler manifold with the Kähler form $\Omega$. It is well known that there exists a convex function $f$ such that $\frac{\partial^{2} f}{\partial x^{i} \partial x^{j}}=\frac{\partial \tau_{i}}{\partial x^{j}}$. Then the Kähler form $\Omega$ can be written as

$$
\begin{aligned}
\Omega & =\sum_{i, j=1}^{n} f_{i j} d x^{i} \wedge \nu^{j}+\sum_{\alpha \in R_{M^{+}}} D_{\alpha}\left(d V^{\alpha} \wedge d W^{\alpha}\right) \\
& =\frac{1}{2}\left[\sum_{i, j=1}^{n} f_{i j} \omega^{i} \wedge \bar{\omega}^{j}+\sum_{\alpha \in R_{M^{+}}} D_{\alpha}\left(\omega^{\alpha} \wedge \bar{\omega}^{\alpha}\right)\right] .
\end{aligned}
$$

We denote $\Omega$ by $\Omega_{f}$. Then the Riemannian metric is given by

$$
\begin{aligned}
\mathcal{G}_{f} & =\sum_{i, j=1}^{n} f_{i j}\left(d x^{i} \otimes d x^{j}+\nu^{i} \otimes \nu^{j}\right)+\sum_{\alpha \in R_{M^{+}}} D_{\alpha}\left(d V^{\alpha} \otimes d V^{\alpha}+d W^{\alpha} \otimes d W^{\alpha}\right) \\
& =\sum_{i, j} \frac{f_{i j}}{2}\left(\omega^{i} \otimes \bar{\omega}^{j}+\bar{\omega}^{j} \otimes \omega^{i}\right)+\sum_{\alpha \in R_{M^{+}}} \frac{D_{\alpha}}{2}\left(\omega^{\alpha} \otimes \bar{\omega}^{\alpha}+\bar{\omega}^{\alpha} \otimes \omega^{\alpha}\right) .
\end{aligned}
$$


Let

$$
\xi_{i}=\frac{\partial f}{\partial x^{i}}, \quad u\left(\xi_{1}, \ldots, \xi_{n}\right)=\sum_{i=1}^{n} x^{i} \xi_{i}-f(x)
$$

Then

$$
\mathcal{G}_{u}=\sum_{i, j=1}^{n}\left(u_{i j} d \xi_{i} \otimes d \xi_{j}+u^{i j} \nu^{i} \otimes \nu^{j}\right)+\sum_{\alpha \in R_{M^{+}}} D_{\alpha}\left(d V^{\alpha} \otimes d V^{\alpha}+d W^{\alpha} \otimes d W^{\alpha}\right),
$$

where $u_{i j}=\frac{\partial^{2} u}{\partial \xi_{i} \partial \xi_{j}}$ and $\left(u^{i j}\right)$ is the inverse matrix of $\left(u_{i j}\right)$. The Kähler form $\Omega$ and the Kähler metric $\mathcal{G}$ can be extended over $G \times_{K} M$.

Suppose that $\Delta$ is defined by linear inequalities $h_{k}(\xi)-c_{k}>0$, for $k=1, \cdots, d$, where $c_{k}$ are constants and $h_{k}$ are affine linear functions in $\mathbb{R}^{n}, k=1, \cdots, d$, and each $h_{k}(\xi)-c_{k}=0$ defines a facet of $\Delta$. Write $\delta_{k}(\xi)=h_{k}(\xi)-c_{k}$ and set

$$
v(\xi)=\sum_{k} \delta_{k}(\xi) \log \delta_{k}(\xi) \text {. }
$$

It defines a Kähler metric on $G \times_{K} M$, which we call the Guillemin metric.

\section{Generalized Abreu Equations}

In this section we calculate the Ricci tensor and the scaler curvature of $G \times_{K} M$. Podesta and Spiro have calculated the Ricci tensor in [20]. Here we use a different method. First we note that for any $(1,0)$-vector fields $X, Y$, we have $\nabla_{X} Y$ is also a $(1,0)$-vector field. In fact, let $\left(z^{1}, \cdots, z^{n+l}\right)$ be holomorphic coordinates. Then $X=$ $\sum_{i=1}^{n+l} X^{i} \frac{\partial}{\partial z^{i}}$ and $Y=\sum_{i=1}^{n+l} Y^{i} \frac{\partial}{\partial z^{i}}$. Here $X^{i}, Y^{i}$ may not be holomorphic functions. Then

$$
\nabla_{\bar{X}} Y=\sum_{i, j} \bar{X}^{i}\left(Y^{j} \nabla_{\frac{\partial}{\partial \bar{z}^{i}}} \frac{\partial}{\partial z^{j}}+\frac{\partial Y^{j}}{\partial \bar{z}^{i}} \frac{\partial}{\partial z^{j}}\right)=\sum_{i, j} \bar{X}^{i} \frac{\partial Y^{j}}{\partial \bar{z}^{i}} \frac{\partial}{\partial z^{j}} .
$$

We want find a smooth holomorphic $(n, 0)$-field $L\left(\wedge_{j=1}^{n} S_{j}\right) \wedge\left(\wedge_{\alpha \in R_{M^{+}}} S_{\alpha}\right)$ on $\tau^{-1}(\Delta)$, where $L$ is a smooth function on $\tau^{-1}(\Delta)$. By the Koszul formula, for any $j \leq n, \alpha \in$ $R_{M^{+}}$, we have

$$
\begin{aligned}
& g\left(\nabla_{\bar{S}_{j}} S_{\alpha}, \bar{S}_{\alpha}\right) \\
& =\frac{1}{2}\left[\bar{S}_{j} g\left(S_{\alpha}, \bar{S}_{\alpha}\right)-g\left(\bar{S}_{j},\left[S_{\alpha}, \bar{S}_{\alpha}\right]\right)+g\left(S_{\alpha},\left[\bar{S}_{\alpha}, \bar{S}_{j}\right]\right)+g\left(\bar{S}_{\alpha},\left[\bar{S}_{j}, S_{\alpha}\right]\right)\right] \\
& =\frac{1}{2}\left[\frac{1}{4} \frac{\partial D_{\alpha}}{\partial x^{j}}-g\left(\bar{S}_{j},-\sqrt{-1} \sum_{k=1}^{n} M_{\alpha}^{k} H_{k}\right)\right]+\frac{1}{4} D_{\alpha} \alpha\left(\tilde{h}_{j}\right)=\frac{1}{4} D_{\alpha} \alpha\left(\tilde{h}_{j}\right) .
\end{aligned}
$$

Similarly we have for any $\alpha, \beta \in R_{M^{+}}, j, k, l \leq n$,

$$
g\left(\nabla_{\bar{S}_{j}} S_{l}, \bar{S}_{k}\right)=g\left(\nabla_{\bar{S}_{\alpha}} S_{l}, \bar{S}_{k}\right)=g\left(\nabla_{\bar{S}_{\alpha}} S_{\beta}, \bar{S}_{\beta}\right)=0 .
$$


Denote $\mathbb{T}=\left(\wedge_{j=1}^{n} S_{j}\right) \wedge\left(\wedge_{\alpha \in R_{M^{+}}} S_{\alpha}\right)$. By (4.1) and (4.2), it is easy to see that

$$
\nabla_{\bar{S}_{\beta}} \mathbb{T}=0, \quad \nabla_{\bar{S}_{j}} \mathbb{T}=\left(\sum_{\alpha \in R_{M^{+}}} \frac{\alpha\left(\tilde{h}_{j}\right)}{2}\right) \mathbb{T} .
$$

Denote $\sigma_{i}=2 \sum_{\alpha \in R_{M^{+}}} \alpha\left(\tilde{h}_{i}\right)$. Choose $L=e^{-\frac{\sum_{i=1}^{n} \sigma_{i} x^{i}}{2}}$, then

$$
\nabla_{\bar{S}_{\beta}}(L \mathbb{T})=0, \quad \nabla_{\bar{S}_{i}}(L \mathbb{T})=0, \quad i \leq n, \alpha \in R_{M^{+}} .
$$

It follows that

$$
e^{\sum_{i=1}^{n} \sigma_{i} x^{i}}\left(\wedge_{j=1}^{n} \omega^{j}\right) \bigwedge\left(\wedge^{\alpha \in R_{M+}} \omega_{\alpha}\right)=|h|^{2}\left(d z^{i} \wedge d \bar{z}^{i}\right)^{n+l},
$$

for some holomorphic function $h$. Then we have

$$
\Omega^{n+l}=\operatorname{det}\left(\frac{\partial^{2} f}{\partial x^{i} \partial x^{j}}\right) \mathbb{D} e^{-\sum_{i=1}^{n} \sigma_{i} x^{i}}|h|^{2}\left(\sum d z^{i} \wedge d \bar{z}^{i}\right)^{n+l} .
$$

Denote $\mathbb{F}_{\Delta}=\operatorname{det}\left(f_{i j}\right) \mathbb{D}$. Choose $\left\{S_{i}, \bar{S}_{i}, S_{\alpha}, \bar{S}_{\alpha}, i \leq n, \alpha \in R_{M^{+}}\right\}$as a local frame field. The Ricci tensor can be written as

$$
\sum_{A, B} \operatorname{Ric}\left(\frac{\partial}{\partial z^{A}}, \frac{\partial}{\partial \bar{z}^{B}}\right) d z^{A} \wedge d \bar{z}^{B}=-\sum_{A, B}\left[\log \mathbb{F}_{\Delta}-\sum_{i=1}^{n} \sigma_{i} x^{i}\right]_{, A \bar{B}} \omega^{A} \wedge \bar{\omega}^{B} .
$$

where "," denotes the covariant derivatives of $\mathcal{G}_{f}$ with respect to this frame field.

Since $\nabla_{\bar{S}_{A}} S_{B}$ is $(1,0)$-vector field and $\mathcal{G}\left(S_{\beta}, \bar{S}_{l}\right)=0$, by Koszul formula, we have

$$
\begin{aligned}
& \mathcal{G}\left(\nabla_{\bar{S}_{\alpha}} S_{\beta}, \frac{\partial}{\partial x^{l}}\right)=\mathcal{G}\left(\nabla_{\bar{S}_{\alpha}} S_{\beta}, \bar{S}_{l}\right) \\
& =\frac{1}{2}\left[-\bar{S}_{l} \mathcal{G}\left(\bar{S}_{\alpha}, S_{\beta}\right)-\mathcal{G}\left(\bar{S}_{\alpha},\left[S_{\beta}, \bar{S}_{l}\right]\right)+\mathcal{G}\left(S_{\beta},\left[\bar{S}_{l}, \bar{S}_{\alpha}\right]\right)+\mathcal{G}\left(\bar{S}_{l},\left[\bar{S}_{\alpha}, S_{\beta}\right]\right)\right] \\
& =\frac{1}{2} \delta_{\alpha \beta}\left[-\frac{1}{4} \frac{\partial D_{\alpha}}{\partial x^{l}}-\sum_{j} \frac{f_{j l}}{2} M_{\alpha}^{j}\right]=-\frac{1}{4} \frac{\partial D_{\alpha}}{\partial x^{l}} \delta_{\alpha \beta} .
\end{aligned}
$$

where we used $\frac{\partial D_{\alpha}}{\partial x^{l}}=\sum \frac{\partial D_{\alpha}}{\partial \xi_{k}} \frac{\partial \xi_{k}}{\partial x^{l}}=2 \sum_{k} M_{\alpha}^{k} f_{k l}$. Similarly we have

$$
\mathcal{G}\left(\nabla_{\bar{S}_{j}} S_{k}, \frac{\partial}{\partial x^{l}}\right)=\mathcal{G}\left(\nabla_{\bar{S}_{j}} S_{\alpha}, \frac{\partial}{\partial x^{l}}\right)=\mathcal{G}\left(\nabla_{\bar{S}_{\alpha}} S_{j}, \frac{\partial}{\partial x^{l}}\right)=0
$$

For any function $F$ depending only on $\left(x^{1}, \cdots, x^{n}\right)$, we have

$$
F_{, A \bar{B}}=S_{A} \bar{S}_{B} F-\mathcal{G}\left(\nabla_{S_{A}} \bar{S}_{B}, \frac{\partial}{\partial x^{l}}\right) f^{k l} \frac{\partial F}{\partial x^{k}} .
$$

Then using (4.4) and (4.5) we have

$$
F_{, j \bar{k}}=\frac{1}{4} \frac{\partial^{2} F}{\partial x^{j} \partial x^{k}}, \quad F_{, \alpha \bar{j}}=0, \quad F_{, j \bar{\alpha}}=0 \quad F_{, \alpha \bar{\beta}}=\delta_{\alpha \beta} \frac{1}{4} \sum f^{k l} \frac{\partial D_{\alpha}}{\partial x^{k}} \frac{\partial F}{\partial x^{l}} .
$$


By (4.3) and (4.6), the Ricci curvatures are given by

$$
\begin{aligned}
& \operatorname{Ric}\left(S_{j}, \bar{S}_{k}\right)=-\frac{1}{4} \frac{\partial^{2} \log \mathbb{F}_{\Delta}}{\partial x^{j} \partial x^{k}}, \quad \operatorname{Ric}\left(S_{\alpha}, \bar{S}_{k}\right)=\operatorname{Ric}\left(S_{j}, \bar{S}_{\alpha}\right)=0, \\
& \operatorname{Ric}\left(S_{\alpha}, \bar{S}_{\beta}\right)=\delta_{\alpha \beta}\left[-\frac{1}{4} \sum f^{k l} \frac{\partial D_{\alpha}}{\partial x^{k}} \frac{\partial \log \mathbb{F}_{\Delta}}{\partial x^{l}}+\frac{1}{4} \sum \frac{\partial D_{\alpha}}{\partial \xi_{k}} \sigma_{k}\right],
\end{aligned}
$$

The scalar curvature can be written as

$$
\mathbb{S}=-\sum_{i, j} f^{i j} \frac{\partial^{2} \log \mathbb{F}_{\Delta}}{\partial x^{i} \partial x^{j}}-\sum_{k, l} f^{k l} \frac{\partial \log \mathbb{D}}{\partial x^{k}} \frac{\partial \log \mathbb{F}_{\Delta}}{\partial x^{l}}+h_{G}
$$

In terms of $\xi$ and $u(\xi), \mathbb{S}$ can be written as

$$
\mathbb{S}=-\frac{1}{\mathbb{D}} \sum_{i, j=1}^{n} \frac{\partial^{2} \mathbb{D} u^{i j}}{\partial \xi_{i} \partial \xi_{j}}+h_{G}
$$

Here

$$
\mathbb{D}=\prod_{\alpha \in R_{M^{+}}} D_{\alpha}, \quad h_{G}=\sum \sigma_{i} \frac{\partial \log \mathbb{D}}{\partial \xi_{i}}=\sum_{i, j} f^{i j} \sigma_{i} \frac{\partial \log \mathbb{D}}{\partial x^{j}},
$$

where $\sigma$ is the sum of the positive roots of $R_{M}^{+}$, and

$$
\sigma_{i}=-2 \sum_{\alpha \in R_{M}^{+}} \alpha\left(\sqrt{-1} \tilde{H}_{i}\right)
$$

Put $A=\mathbb{S}-h_{G}$. We will consider the PDE

$$
-\frac{1}{\mathbb{D}} \sum_{i, j=1}^{n} \frac{\partial^{2} \mathbb{D} u^{i j}}{\partial \xi_{i} \partial \xi_{j}}=A .
$$

The equation (4.11) was introduced by Donaldson [15] in the study of the scalar curvature of toric fibration, see also [21] and [19]. We call (4.11) a generalized Abreu Equation.

\section{UNIFORM STABILITY}

We introduce several classes of functions. Set

$$
\begin{aligned}
& \mathcal{C}=\{u \in C(\bar{\Delta}): u \text { is convex on } \bar{\Delta} \text { and smooth on } \Delta\}, \\
& \mathbf{S}=\{u \in C(\bar{\Delta}): u \text { is convex on } \bar{\Delta} \text { and } u-v \text { is smooth on } \bar{\Delta}\},
\end{aligned}
$$

where $v$ is given in (3.9). For a fixed point $p_{o} \in \Delta$, we consider

$$
\begin{aligned}
& \mathcal{C}_{p_{o}}=\left\{u \in \mathcal{C}: u \geq u\left(p_{o}\right)=0\right\}, \\
& \mathbf{S}_{p_{o}}=\left\{u \in \mathbf{S}: u \geq u\left(p_{o}\right)=0\right\} .
\end{aligned}
$$

We say functions in $\mathcal{C}_{p_{o}}$ and $\mathbf{S}_{p_{o}}$ are normalized at $p_{o}$. 
We consider the generalized Abreu equation (4.11), where, $\mathbb{D}>0$ and $A$ are given smooth functions on $\bar{\Delta}$. Following [19] we consider the functional

$$
\mathcal{F}_{A}(u)=-\int_{\Delta} \log \operatorname{det}\left(u_{i j}\right) \mathbb{D} d \mu+\mathcal{L}_{A}(u)
$$

where

$$
\mathcal{L}_{A}(u)=\int_{\partial \Delta} u \mathbb{D} d \sigma-\int_{\Delta} A u \mathbb{D} d \mu .
$$

$\mathcal{F}_{A}$ is called the Mabuchi functional and $\mathcal{L}_{A}$ is closely related to the Futaki invariants. The Euler-Lagrangian equation for $\mathcal{F}_{A}$ is (4.11). It is known that, if $u \in \mathbf{S}$ satisfies the equation (4.11), then $u$ is an absolute minimizer for $\mathcal{F}_{A}$ on $\mathbf{S}$.

Definition 5.1. Let $\mathbb{D}>0$ and $A$ be smooth functions on $\bar{\Delta}$. Then, $(\Delta, \mathbb{D}, A)$ is called uniformly $K$-stable if the functional $\mathcal{L}_{A}$ vanishes on affine-linear functions and there exists a constant $\lambda>0$ such that, for any $u \in \mathcal{C}_{p_{o}}$,

$$
\mathcal{L}_{A}(u) \geq \lambda \int_{\partial \Delta} u \mathbb{D} d \sigma
$$

We also say that $\Delta$ is $(\mathbb{D}, A, \lambda)$-stable.

Using the same method in [10] we immediately get

Theorem 5.2. If the equation (4.11) has a solution in $\mathrm{S}$, then $(\Delta, \mathbb{D}, A)$ is uniform $K$ stable.

Donaldson [14] derived a $L^{\infty}$ estimate for the Abreu's equation in $\Delta \subset \mathbb{R}^{2}$. His method can be applied directly to the generalized Abreu's Equation $\Delta \subset \mathbb{R}^{2}$ ( see also [19]). We have

Theorem 5.3. Let $\Delta \subset \mathbb{R}^{2}$ be a Delzant polytope, $\mathbb{D}>0$ and $A$ be two smooth functions defined on $\bar{\Delta}$. Let $u \in C^{\infty}(\Delta)$ satisfying (4.11). Suppose that $\Delta$ is $(\mathbb{D}, A, \lambda)$-stable. Then there is a constant $\mathrm{C}_{o}>0$, depending on $\lambda, \Delta, \mathbb{D}$ and $\|A\|_{C^{0}}$, such that $\mid \max _{\overline{\bar{A}}} u-$ $\min _{\bar{\Delta}} u \mid \leq \mathrm{C}_{o}$

\section{DifFEREnTIAL INEQUALities}

We first introduce some notations. Let $p$ be a vertex of $\Delta$, the edges meeting $p$ are the form $p+t E_{i}, t \geq 0, E^{i} \in \mathbb{Z}^{n}, i=1, \ldots, n$. Consider the base $\left\{p ; E^{1}, \ldots, E^{n}\right\}$, let $\xi^{p}=\left(\xi_{1}^{p}, \ldots, \xi_{n}^{p}\right)$ be the coordinates with respect to the base $\left\{p ; E^{i}\right\}$. Suppose that

$$
p=\sum_{i=1}^{n} c_{i} \nu^{i}, \quad \nu^{i}=\sum_{i=1}^{n} a_{j}^{i} E^{j}, \quad\left(a_{i}^{j}\right) \in S L(n, \mathbb{Z}),
$$


we have the coordinate transformation

$$
\xi_{i}^{p}=\sum_{j=1}^{n} a_{i}^{j}\left(\xi_{j}-c_{i}\right), \quad \xi^{p}(p)=0, \quad \Delta \subset\left\{\xi^{p} \mid \xi_{i}^{p}>0\right\} .
$$

Set

$$
x_{p}^{i}=\frac{\partial u}{\partial \xi_{i}^{p}}, \quad f_{p}\left(x_{p}^{1}, \ldots, x_{p}^{n}\right)=\sum x_{p}^{i} \xi_{i}^{p}-u .
$$
Let

Then $f_{p}$ can be naturally extend to a smooth function on a neighborhood $U_{p}$ of $\tau_{M}^{-1}(p)$.

$$
w^{i}=x_{p}^{i}+\sqrt{-1} y_{p}^{i}, \quad z_{p}^{i}=e^{\frac{w_{p}^{i}}{2}}, \quad i=1, \cdots, n .
$$

Then $\left(z_{p}^{1}, \cdots, z_{p}^{n}\right)$ is a local holomorphic coordinates of $U_{p}$. We have (see [8])

$$
f_{p}=f-\sum_{i=1}^{n} c_{i} x^{i}
$$

Denote

$$
\begin{aligned}
& \mathbb{F}_{p}=4^{2 n} \operatorname{det}\left(\frac{\partial^{2} f_{p}}{\partial z_{p}^{i} \partial \bar{z}_{p}^{j}}\right) \mathbb{D}=\operatorname{det}\left(\frac{\partial^{2} f_{p}}{\partial x_{p}^{i} \partial x_{p}^{j}}\right) e^{-x_{p}^{1}-x_{p}^{2}-\ldots-x_{p}^{n}} \mathbb{D} . \\
& \Psi_{p}:=\left\|\nabla \log \mathbb{F}_{p}\right\|_{f}^{2}, \quad P=\exp \left(\kappa \mathbb{F}_{p}^{a}\right) \sqrt{\mathbb{F}_{p}} \Psi_{p},
\end{aligned}
$$

where $a$ and $\kappa$ are positive constants to be determined later.

More general, for each $(n-k)$-dimensional face of $\Delta$, one can associate it a complex coordinate chart of $M$ :

$$
\mathrm{U}_{k} \cong \mathbb{C}^{k} \times\left(\mathbb{C}^{*}\right)^{n-k}
$$

Let $\left(w^{1}, \cdots, w^{n}\right), w^{i}=x^{i}+\sqrt{-1} y^{i}$, be the log-affine coordinate of $M$. Set

$$
z^{i}=e^{\frac{w^{i}}{2}}, \quad i \leq k
$$

Then $\left(z^{1}, \ldots, z^{k}, w^{k+1}, \ldots, w^{n}\right)$ is local holomorphic coordinates of $\mathbf{U}_{k}$. Denote

$$
Z_{n-k}=\left\{p \in M \mid z^{i}=0, i \leq k\right\}, \quad E=\left\{\xi \mid \xi_{i}=0, i \leq k\right\}
$$

where $\xi_{i}=\frac{\partial f}{\partial x^{i}}$. Let $f_{E}=f-\sum_{i=1}^{k} c_{i} x^{i}-d$, where $c_{i}, d$ are constants such that $\tau_{f_{E}}\left(Z_{n-k}\right)=E$. Then $f_{E}$ can be naturally extend to a smooth function in $\bigcup_{k}$. Denote

$$
\begin{aligned}
& \mathbb{F}_{E}=\operatorname{det}\left(\frac{\partial^{2} f_{E}}{\partial x^{i} \partial x^{j}}\right) e^{-\sum_{i=1}^{k} x^{i}} \mathbb{D} . \\
& \boldsymbol{\Psi}_{E}:=\left\|\nabla \log \mathbb{F}_{E}\right\|_{f}^{2}, \quad P_{E}=\exp \left(\kappa \mathbb{F}_{E}^{a}\right) \sqrt{\mathbb{F}_{E}} \Psi_{E} .
\end{aligned}
$$

Set $V_{E}=\log \mathbb{F}_{E}, \mathbb{A}_{E}=-\square \log \mathbb{F}_{E}$. 
Let $\Omega_{g}$ be the Guillemin metric on $G \times_{K} M$ with local potential function $g$. Denote by $\dot{R}_{i \bar{j} k \bar{l}}$ and $\dot{R}_{i \bar{j}}$ the curvature tensor and the Ricci curvature of $\Omega_{g}$, respectively. Put

$$
\dot{\mathcal{R}}:=\sqrt{\sum g^{m \bar{n}} g^{k \bar{l}} g^{i \bar{j}} g^{s \bar{t}} \dot{R}_{m \bar{l} \bar{t}} \dot{R}_{k \bar{n} s \bar{j}}}
$$

Let $\Omega_{f}$ be a Kähler metric on $G \times_{K} M$ in the same Kähler class as $\Omega_{g}$ with local potential function $f$. Then there is a global defined function $\phi \in C^{\infty}\left(G \times_{K} M\right)$ such that $\phi=$ $f-g$. Denote $\square=\sum f^{A \bar{B}} \frac{\partial^{2}}{\partial z^{A} \partial \bar{z}^{B}}$ the Laplacian operator on $G \times_{K} M$ with respect to the metric $\Omega_{f}$. Obviously $n-\square \phi=\sum f^{i \bar{j}} g_{i \bar{j}}:=T$. Put

$$
Q=e^{-\mathrm{N}_{1}(\phi-\inf \phi+1)} \sqrt{\mathbb{F}_{p}} T,
$$

where $\mathrm{N}_{1}>0$ is a constant.

In this section we establish some differential inequalities in $G\left(U_{p}\right)$ for $\log \mathbb{F}, P$ and $Q$.

6.1. Subharmonic function $\log \mathbb{F}_{p}+N f_{p}$. For any function $F$ depending only on $\left(x^{1}, \cdots, x^{n}\right)$ we have

$$
\square F=\sum f^{i j} F_{i j}+\sum f^{i j} \frac{\log \mathbb{D}}{\partial x^{i}} F_{j}=\sum f^{i j} F_{i j}+\sum \frac{\log \mathbb{D}}{\partial \xi_{j}} F_{j} .
$$

where $F_{i}=\frac{\partial F}{\partial x^{i}}, F_{i j}=\frac{\partial^{2} F}{\partial x^{i} \partial x^{j}}$.

Lemma 6.1. Choose $\left\{o, \nu^{i}, i=1, \ldots, n\right\}$ as a base of $\mathbb{R}^{n}$, let $\bar{\Delta} \subset\left\{\left(\xi_{1}, \ldots, \xi_{n}\right) \mid \xi_{1}>\right.$ $\left.0, \xi_{2}>0, \ldots, \xi_{n}>0\right\}$ be a Delzant polytope satisfying

$$
\sum_{\alpha \in R_{M^{+}}} \frac{\sum_{j=1}^{n} M_{\alpha}^{j} \operatorname{diam}(\Delta)}{D_{\alpha}}<\frac{n}{4} .
$$

Then there is a constant $N>0$ depending only on $n, \mathbb{D}, \Delta$ and the position of $\Delta$ in $\mathbb{R}^{n}$ such that for any vertex $p$ of $\Delta$

$$
\square\left(\log \mathbb{F}_{p}+N f_{p}\right)>0
$$

Proof. By 6.1 we have

$$
\begin{aligned}
\square f_{p} & =\square\left(f-\sum c_{i} x^{i}\right)=n+\sum \frac{\partial \log \mathbb{D}}{\partial \xi_{i}}\left(\frac{\partial f}{\partial x^{i}}-c_{i}\right) \\
& \geq n-\sum_{\alpha \in R_{M^{+}}} \frac{\sum_{j} M_{\alpha}^{j} \operatorname{diam}(\Delta)}{\sum_{j} M_{\alpha}^{j} \xi_{j}} \geq \frac{n}{2} .
\end{aligned}
$$


As $x^{i}=\frac{\partial u}{\partial \xi_{i}}=\sum_{j} \frac{\partial u}{\partial \xi_{j}^{p}} \frac{\partial \xi_{j}^{p}}{\partial \xi_{i}}=\sum a_{i}^{j} x_{p}^{j}$, we have $\operatorname{det}\left(\frac{\partial^{2} f}{\partial x_{p}^{i} \partial x_{p}^{j}}\right)=\operatorname{det}\left(\frac{\partial^{2} f}{\partial x^{i} \partial x^{j}}\right)$. Denote by $\left(b_{i}^{j}\right)$ the inverse of $\left(a_{i}^{j}\right)$. Then by (4.10) and (6.6)

$$
\square \log \mathbb{F}_{p}=-\mathbb{S}+\sum_{k=1}^{m} \frac{\partial \log \mathbb{D}}{\partial \xi_{k}^{p}}\left(\sigma_{k}-\sum_{j} b_{k}^{j}\right):=-\mathbb{A}_{p} .
$$

Denote $\mathcal{P}$ be the set of all vertices of $\bar{\Delta}$. Let $N$ be the constant such that

$$
\max _{p \in \mathcal{P}} \max _{\xi \in \bar{\Delta}} \mathbb{A}_{p}<\frac{n}{2} N
$$

Then for any $p \in \mathcal{P}$, we have

$$
\square\left(\log \mathbb{F}_{p}+N f_{p}\right)>0
$$

6.2. Differential inequalities for $P$. We are going to calculate $\square P$ and derive a differential inequality. This inequality is similar to Lemma 5.1 in [7]. In this subsection we denote $\mathbb{F}_{E}, \mathbb{A}_{E}, \Psi_{E}, \cdots$ by $\mathbb{F}, \mathbb{A}, \Psi, \cdots$, etc. By (4.6), (4.7) and (4.8) we have

$$
\begin{aligned}
& (\log \mathbb{F})_{, j \bar{k}}=-\operatorname{Ric}\left(S_{j}, \bar{S}_{k}\right), \quad(\log \mathbb{F})_{, \alpha \bar{k}}=0, \quad(\log \mathbb{F})_{, k \bar{\alpha}}=0, \\
& (\log \mathbb{F})_{, \alpha \bar{\beta}}=-\operatorname{Ric}\left(S_{\alpha}, \bar{S}_{\beta}\right)+\frac{\delta_{\alpha \beta}}{4} h_{\alpha},
\end{aligned}
$$

where $h_{\alpha}=\sum_{k} \frac{\partial D_{\alpha}}{\partial \xi_{k}}\left(\sigma_{k}-\sum_{j} c_{k}^{j}\right)$ and $c_{k}^{j}$ are constants depending only on $E$. Denote by $f_{, A \bar{B}}$ the components of the metric $\mathcal{G}$ with respect to the frame $\left\{S_{A}, \bar{S}_{B}\right\}$, and by $\left(f^{A \bar{B}}\right)$ the inverse of $\left(f_{, A \bar{B}}\right)$. Put $W=\operatorname{det}\left(f_{, i \bar{j}}\right), V=\log \mathbb{F}$,

$$
\left\|V_{, A \bar{B}}\right\|_{f}^{2}=\sum f^{A \bar{B}} f^{C \bar{D}} V_{A \bar{D}} V_{B \bar{C}}, \quad\left\|V_{, A B}\right\|_{f}^{2}=\sum f^{A \bar{B}} f^{C \bar{D}} V_{, A C} V_{, \bar{B} \bar{D}}
$$

\section{Lemma 6.2.}

$$
\frac{\square P}{P} \geq \frac{\left\|V_{, A \bar{B}}\right\|_{f}^{2}}{2 \boldsymbol{\Psi}}+a^{2} \kappa\left(1-2 \kappa \mathbb{F}^{a}\right) \mathbb{F}^{a} \boldsymbol{\Psi}-\frac{2|\langle\nabla \mathbb{A}, \nabla V\rangle|}{\boldsymbol{\Psi}}-\left(a \kappa \mathbb{F}^{a}+\frac{1}{2}\right) \mathbb{A},
$$

where $\langle$,$\rangle denotes the inner product with respect to the metric \Omega_{f}$.

Proof. By definition,

$$
\square \boldsymbol{\Psi}=\sum f^{A \bar{B}} f^{C \bar{D}}\left(V_{, A} V_{, \bar{B} C \bar{D}}+V_{, A C \bar{D}} V_{, \bar{B}}+V_{, A C} V_{, \bar{B} \bar{D}}+V_{, A \bar{D}} V_{, \bar{B} C}\right) .
$$

Since $V$ depends only on $\left(x^{1}, \cdots, x^{n}\right)$, we have

$$
V_{, \alpha}=V_{, \bar{\alpha}}=0 \text {. }
$$

By the Ricci identities, 6.12) and $f^{j \bar{\alpha}}=0$, we have

$$
V_{, \bar{B} C \bar{D}}=V_{, C \bar{D} \bar{B}}, \quad V_{, A C \bar{D}}=V_{, C \bar{D} A}+\sum f^{j \bar{h}} V_{, j} R_{C \bar{h} A \bar{D}} .
$$


It follows that

$$
\square \boldsymbol{\Psi}=\left\|V_{, A B}\right\|^{2}+\left\|V_{A \bar{B}}\right\|^{2}+\sum f^{i \bar{j}} f^{k \bar{l}}\left(-V_{i \bar{l}} V_{, k} V_{, \bar{j}}\right)-2 R e\left(\sum f^{i \bar{j}} V_{, i} \mathbb{A}_{, \bar{j}}\right),
$$

where we use the facts $R_{i \bar{j}}=-V_{k \bar{l}}$ and $\square V=-\mathbb{A}$. Denote $\Pi=a \kappa \mathbb{F}^{a}+\frac{1}{2}$. Then

$$
\begin{aligned}
P_{, i} & =P\left(\frac{\boldsymbol{\Psi}_{, i}}{\boldsymbol{\Psi}}+\Pi V_{, i}\right)=: P \Lambda_{i} \\
\square P & =P\left[\sum f^{i \bar{j}} \Lambda_{i} \Lambda_{\bar{j}}+\frac{\square \boldsymbol{\Psi}}{\boldsymbol{\Psi}}-\frac{\|\nabla \boldsymbol{\Psi}\|_{f}^{2}}{\boldsymbol{\Psi}^{2}}+\Pi \square V+a^{2} \kappa \mathbb{F}^{a} \boldsymbol{\Psi}\right] .
\end{aligned}
$$

For any point $q \in G\left(\mathrm{U}_{k}\right)$, we choose an affine transformation of the frame fields $\left\{S_{A}, \bar{S}_{B}\right\}$ such that, at $q$,

$$
f_{, i \bar{j}}=c \delta_{i j}, \quad 1 \leq i, j \leq n, \quad V_{1}=V_{\overline{1}}, \quad V_{i}=V_{\bar{i}}=0, \quad \forall 1 \leq i \leq n,
$$

where $c=[W(q)]^{\frac{1}{n}}$. Then by the same arguments of Lemma 5.1 in [7] we can prove the lemma.

6.3. Differential inequality for $Q$. The following differential inequality of $n-\square \phi$ has been proved in [7] (see Section $\S 5$, Inequality-II)

\section{Lemma 6.3.}

$$
\square \log (n-\square \phi) \geq-\|\operatorname{Ric}\|_{f}-\dot{\mathcal{R}}(n-\square \phi)
$$

In the following we restrict ourself to $n=2$.

\section{Lemma 6.4. Suppose that}

$$
\left\|\mathbb{A}_{p}\right\|_{C^{1}\left(\tau_{f}\left(U_{p}\right)\right)} \leq \mathrm{N}_{2}, \quad \max _{\bar{U}_{p}} \mathbb{F} \leq \mathrm{N}_{2}, \quad \max _{\bar{U}_{p}}|\phi|+|z| \leq \mathrm{N}_{2}
$$

for some constant $\mathrm{N}_{2}>0$. Then we may choose

$$
\mathrm{N}_{1}=100, a=\frac{1}{3}, \kappa=\left[4 \mathrm{~N}_{2}^{\frac{1}{3}}\right]^{-1}
$$

such that

$$
\square\left(P+Q+\mathrm{C}_{1} f_{p}\right) \geq \mathrm{C}_{2}(P+Q)^{2}>0
$$

for some positive constants $\mathrm{C}_{1}$ and $\mathrm{C}_{2}$ that depend only on $\mathrm{N}_{2}$, the structure constants of $\mathfrak{g}, \mathbb{D}, \Delta$ and the position of $\Delta$ in $\mathbb{R}^{2}$.

Proof. Applying Lemma 6.2 and the choice of $a=\frac{1}{3}$ and $\kappa$, in particular, $\kappa \mathbb{F}^{a} \leq 1 / 4$, we have

$$
\frac{\boldsymbol{\Psi} \square P}{P} \geq\left(\frac{1}{2}\left\|V_{, A \bar{B}}\right\|_{f}^{2}+\frac{1}{18} \kappa \mathbb{F}^{\frac{1}{3}} \boldsymbol{\Psi}^{2}\right)-(2|\langle\nabla \mathbb{A}, \nabla V\rangle|+\boldsymbol{\Psi}|\mathbb{A}|) .
$$


Treatment for $\langle\nabla \mathbb{A}, \nabla V\rangle$ : using log-affine coordinates we have

$$
|\langle\nabla \mathbb{A}, \nabla V\rangle|=\left|\sum f^{i j} \frac{\partial \mathbb{A}}{\partial x^{i}} \frac{\partial V}{\partial x^{j}}\right|=\left|\sum \frac{\partial K}{\partial \xi_{k}} \frac{\partial V}{\partial x^{k}}\right| \leq \mathrm{N}_{2} \sum_{j}\left|\frac{\partial V}{\partial x^{j}}\right| .
$$

If we use the complex coordinates $z_{i}$, we have

$$
\left|\frac{\partial V}{\partial x^{j}}\right|=\left|z_{j} \frac{\partial V}{\partial z_{j}}\right|
$$

Since $|z|$ is bounded, in this coordinates we have

$$
C^{-1} \leq g_{i \bar{j}} \leq C, \quad \sum f^{i \bar{i}} \leq C \sum f^{i \bar{j}} g_{i \bar{j}} \leq C T .
$$

Then we conclude that

$$
|\langle\nabla \mathbb{A}, \nabla V\rangle| \leq C \sqrt{2 \mathbb{F} T \boldsymbol{\Psi}} .
$$

We explain the last step: suppose that $0<\nu_{1} \leq \nu_{2}$ are the eigenvalues of $\left(f_{i \bar{j}}\right)$, then

$$
\Psi=\sum f^{i \bar{j}} V_{i} V_{\bar{j}} \geq \nu_{2}^{-1}\left(\left|V_{1}\right|^{2}+\left|V_{2}\right|^{2}\right) \geq(\mathbb{F} T)^{-1}\left(\left|V_{1}\right|^{2}+\left|V_{2}\right|^{2}\right) .
$$

Note that

$$
(e \mathbb{F})^{-\frac{1}{2}} \leq \boldsymbol{\Psi} / P=\left(\exp \left(\kappa \mathbb{F}^{\alpha}\right) \mathbb{F}^{\frac{1}{2}}\right)^{-1} \leq \mathbb{F}^{-\frac{1}{2}},
$$

(6.19) is then transformed to be

$$
\square P \geq \mathbb{F}^{\frac{1}{2}}\left(\frac{1}{2}\left\|V_{, A \bar{B}}\right\|_{f}^{2}+\frac{1}{18} \kappa \mathbb{F}^{\frac{1}{3}} \Psi^{2}\right)-C^{\prime} \mathbb{F}^{\frac{1}{2}}(\sqrt{\mathbb{F} T \boldsymbol{\Psi}}+\boldsymbol{\Psi}|\mathbb{A}|) .
$$

Applying the Young inequality and the Schwartz inequality to terms in (6.20), we have that

$$
\square P \geq \frac{1}{2} \mathbb{F}^{\frac{1}{2}}\left\|V_{, A \bar{B}}\right\|_{f}^{2}+C_{1} \mathbb{F}^{-\frac{1}{6}} P^{2}-\epsilon Q T-C_{2}(\epsilon) .
$$

By (6.10), (6.11) and Cauchy inequalities, for any $\delta \in(0,1)$, we obtain that

$$
\|R i c\|_{\mathcal{G}_{f}}^{2}=\sum_{i, j}\left\|V_{i \bar{j}}\right\|^{2}+\sum_{\alpha \in R_{M^{+}}}\left\|V_{, \alpha \bar{\alpha}}+h_{\alpha}\right\|^{2} \leq(1+\delta)\left\|V_{, A \bar{B}}\right\|^{2}+C_{\delta, R_{M^{+}}},
$$

where $C_{\delta, R_{M^{+}}}>0$ is a constant depending only on $1 / \delta$ and $\sum_{\alpha \in R_{M^{+}}} h_{\alpha}^{2}$. By a direct calculation and the formula (6.15) we have

$$
\begin{aligned}
\square Q & \geq Q\left(N_{1} T+\frac{1}{2} \square V+\square \log T\right) \\
& \geq Q\left(\left(N_{1}-\dot{\mathcal{R}}\right) T-\frac{1}{2} \mathbb{A}-(1+\delta)\left\|V_{, A \bar{B}}\right\|_{f}-C_{\delta, R_{M^{+}}}\right),
\end{aligned}
$$


Choosing $\delta$ small, using the explicit value $N_{1}$ and the bounds of $\mathbb{F}$ and $\mathbb{A}$, applying the Schwartz inequality properly, we can get

$$
\square Q \geq-\frac{1}{4} \mathbb{F}^{\frac{1}{2}}\left\|V_{, A \bar{B}}\right\|_{f}^{2}+\frac{\mathrm{N}_{1}}{3} Q T-C_{3}\left(\mathrm{~N}_{1}, \mathrm{~N}_{2}, \delta, R_{M^{+}}\right) .
$$

Combining (6.21) and (6.22), and choosing $\epsilon=\frac{1}{100}$, we have

$$
\square(Q+P) \geq C_{1} \mathbb{F}^{-\frac{1}{6}} P^{2}+\frac{N_{1}}{4} Q T-C_{4} .
$$

Note that

$$
T=e^{-N_{1} \phi} \mathbb{F}^{-\frac{1}{2}} Q \geq e^{-N_{1} \phi} \mathbf{N}_{2}^{-\frac{1}{3}} \mathbb{F}^{-\frac{1}{6}} Q \geq C_{5} \mathbb{F}^{-\frac{1}{6}} Q
$$

we get

$$
\square(Q+P) \geq C_{6} \mathbb{F}^{-\frac{1}{6}}(Q+P)^{2}-C_{7}
$$

where $C_{6}, C_{7}$ are constants depending only on $C_{1}, \mathrm{~N}_{1}$ and $\mathrm{N}_{2}$. Our lemma follows from $\square f_{p} \geq n / 2$. and $|\mathbb{F}| \leq \mathrm{N}_{2}$.

6.4. Interior estimate of $\Psi$. We use Lemma 6.2 to derive the interior estimate of $\Psi$ in a geodesic ball in $\bigcup_{k}$. In this subsection we denote $\mathbb{F}_{E}, \mathbb{A}_{E}, \Psi_{E}, \cdots$ by $\mathbb{F}, \mathbb{A}, \Psi, \cdots$, etc.

Lemma 6.5. Let $B_{a}(o) \subset \mathrm{U}_{k}$ be a closed geodesic ball of radius a centered at o with respect to the metric $\mathcal{G}_{f}$. Set $\mathbb{F}_{\diamond}:=\max _{B_{a}(o)} \mathbb{F}$. Suppose that

$$
\min _{B_{a}(o)}|\mathbb{A}| \neq 0, \text { and, } \mathbb{F}^{\frac{1}{2}}\left(\mathcal{K}+\mathbb{K}+\|\nabla \log |\mathbb{A}|\|_{f}^{2}+\Psi\right) \leq 4,
$$

in $B_{a}(o)$. Then the following estimate holds in $B_{a / 2}(o)$

$$
\mathbb{F}^{\frac{1}{2}} \Psi \leq \mathrm{C}_{6}\left[\mathbb{F}_{\diamond}^{\frac{1}{2}} \max _{B_{a}(o)}|\mathbb{A}|+\mathbb{F}_{\diamond}^{\frac{1}{3}} \max _{B_{a}(o)}|\mathbb{A}|^{\frac{2}{3}}+a^{-1} \mathbb{F}_{\diamond}^{\frac{1}{4}}+a^{-2} \mathbb{F}_{\diamond}^{\frac{1}{2}}\right]
$$

where $\mathrm{C}_{6}$ is a constant depending only on $n$.

Proof. Consider the function

$$
F:=\left(a^{2}-r^{2}\right)^{2} P
$$

defined in $B_{a}(o)$, where $r$ denotes the geodesic distance from $o$ to $z$ with respect to the metric $\mathcal{G}_{f} . F$ attains its supremum at some interior point $q^{*}$. Choose $\kappa=\frac{1}{4 \mathbb{F}_{\diamond} \frac{1}{4}}, \quad \alpha=\frac{1}{4}$, As in [7], using Lemma 6.2 and a direct calculation we have, at $q^{*}$,

$$
\epsilon_{o}\left(\frac{\mathbb{F}}{\mathbb{F}_{\diamond}}\right)^{\frac{1}{4}} \Psi-\frac{2\|\nabla \mathbb{A}\|_{f}}{\sqrt{\boldsymbol{\Psi}}}-\frac{9}{16}|\mathbb{A}|-\frac{24 a^{2}}{\left(a^{2}-r^{2}\right)^{2}}-\frac{4(1+r \square r)}{a^{2}-r^{2}} \leq 0,
$$

where $\epsilon_{o}=\frac{1}{128}$. Denote by $\Gamma$ the geodesic from $o$ to $q^{*}$. To estimate $r \square r$, we consider two cases: 
Case 1. Along $\Gamma$ the following estimate holds

$$
\mathbb{F} \geq \frac{1}{100} \mathbb{F}\left(q^{*}\right)
$$

Case 2. There is a point $q \in \Gamma$ such that $\mathbb{F}(q)<\frac{1}{100} \mathbb{F}\left(q^{*}\right)$. Then there is a point $q_{1} \in \Gamma$ such that

$$
\mathbb{F}\left(q_{1}\right)=\frac{1}{100} \mathbb{F}\left(q^{*}\right), \quad \mathbb{F}(q) \geq \frac{1}{100} \mathbb{F}\left(q^{*}\right), \forall q \in\left[q_{1}, q^{*}\right]
$$

Then by the same argument of [7] for both cases we have estimates (6.24) in $B_{a / 2}(o)$.

\section{REFERENCES}

[1] M. Abreu, Kähler geometry of toric varieties and extremal metrics, Internat. J. Math., 9(1998), 641-651.

[2] V. Apostolov, D. Calderbank, P. Gauduchon, C. Tønnesen-Friedman, Christina W. Extremal Kähler metrics on projective bundles over a curve, Adv. Math. 227 (2011), no. 6, 2385 C2424.

[3] V. Apostolov, G. Maschler, Conformally Kähler, Einstein-Maxwell Geometry, arXiv:1512.06391.

[4] A. Arvanitoyeorgos, An Introduction to Li groups and the Geometry of Homogeneous Spaces, Translated from the 1999 Greek original and revised by the author. Student Mathematical Library, 22. American Mathematical Society, Providence, RI, 2003. xvi+141 pp.

[5] B. Chen, A.-M. Li, L. Sheng, The Abreu equation with degenerated boundary conditions, J. Diff. Equations, 252(2012), 5235-5259.

[6] B. Chen, A.-M. Li, L. Sheng, Interior regularity on the Abreu equation, Acta Mathematica Sinica, 29(2013), 33-38.

[7] B. Chen, A.-M. Li, L. Sheng, Affine techniques on extremal metrics on toric surfaces, arXiv:1008.2606

[8] B. Chen, A.-M. Li, L. Sheng, Extremal metrics on toric surfaces, arXiv:1008.2607.

[9] B. Chen, Q. Han, A.-M. Li, L. Sheng, Interior Estimates for the n-dimensional Abreu's Equation, Advances in mathematics, 251(2014), 35-46

[10] B. Chen, A.-M. Li, L. Sheng, Uniform $K$-stability for extremal metrics on toric varieties, arXiv:1109.5228v2.

[11] S. K. Donaldson, Scalar curvature and stability of toric varieties, J. Diff. Geom., 62(2002), 289-349.

[12] S. K. Donaldson, Interior estimates for solutions of Abreu's equation, Collect. Math., 56(2005), 103-142.

[13] S. K. Donaldson, Extremal metrics on toric surfaces: a continuity method, J. Diff. Geom., 79(2008), 389-432

[14] S. K. Donaldson, Constant scalar curvature metrics on toric surfaces, Geom. Funct. Anal., 19(2009), 83-136.

[15] S. K. Donaldson, Kähler geometry on toric manifolds, and some other manifolds with large symmetry, Handbook of Geometric Analysis, No. 1, International Press, Boston, 2008.

[16] V. Guillemin, Kähler structures on toric varieties, J. Diff. Geom., 40(1994), 285-309.

[17] A.-M. Li, R. Xu, U. Simon, F. Jia, Affine Bernstein Problems and Monge-Ampère Equations, World Scientific, 2010.

[18] A.-M. Li, F. Jia, A Bernstein properties of some fourth order partial differential equations, Result. Math., 56 (2009), 109-139. 
[19] T. Nyberg, Constant Scalar Curvature of Toric Fibrations. PhD thesis.

[20] Podesta, Spiro, Kahler-Ricci solitons on homogeneous toric bundles I, II Arxiv DG/0604070/0604071

[21] A. Raza. Scalar curvature and multiplicity-free actions. PhD thesis.

[22] G. Sziékelyhidi, Extremal metrics and K-stability, Bull. London Math. Soc., 39(2007), 76-84.

[23] G. Tian, Canonical Metrics in Kähler Geometry, Lectures in Mathematics ETH Zurich, Birkhäuser Verlag, Basel, 2000.

[24] S.-T. Yau, Open problems in geometry, Proc. Symposia Pure Math., 54(1993), 1-28.

Department of Mathematics, Sichuan University, Chengdu, 610064, China

E-mail address: anminliscu@126.com

(Sheng) Department of Mathematics Sichuan University Chengdu, 610064, China

E-mail address, Corresponding author: lshengscu@gmail . com

(Zhao) Department of Mathematics Sichuan University Chengdu, 610064, China

E-mail address: gs zhao@scu . edu.cn 